\title{
Comparison of Pediatric and Adult ECG Rhythm Analysis by Automated External Defibrillators during Out-of-Hospital Cardiac Arrest
}

\author{
Vessela Krasteva ${ }^{1}$, Sarah Ménétré ${ }^{2}$, Irena Jekova ${ }^{1}$, Todor Stoyanov ${ }^{1}$, Daniel Jost ${ }^{3}$, Benoit Frattini ${ }^{3}$, \\ Sabine Lemoine ${ }^{3}$, Frédéric Lemoine ${ }^{3}$, Vincent Thomas ${ }^{3}$, Jean-Philippe Didon ${ }^{2}$ \\ ${ }^{1}$ Institute of Biophysics and Biomedical Engineering, Bulgarian Academy of Sciences, Sofia, Bulgaria \\ ${ }^{2}$ Signal Processing, Schiller Médical, Wissembourg, France \\ ${ }^{3}$ Paris Fire Brigade, Paris, France
}

\begin{abstract}
This study validates the performance of a commercial automated external defibrillator (AED) on pediatric outof-hospital cardiac arrest interventions. It shows that the $A E D$ with settings validated for adults does not worsen its performance in children, except for rapid non-shockable rhythms (-2\%). Further, this study compares 14 behavioral ECG characteristics (6 morphological, 4 band-pass QRS filter, 4 spectral) on adult and pediatric rhythms, highlighting significant rhythm-specific changes. Discriminant models trained on adults and tested on pediatrics highlight 6 features with the most powerful AUC $>0.9$ and the most robust AUC(Pediatric-Adult) $>$ 0.025. The design of AED shock advisory algorithm, equally safe for adults and pediatrics is possible if the embedded ECG analysis features fulfil the above criteria.
\end{abstract}

\section{Introduction}

The recent 2015 European Resuscitation Council (ERC) guidelines for pediatric life support [1], require that automated external defibrillators (AEDs) are validated for pediatric uses as they have been originally designed for adult patients. In order to guarantee that AEDs are safely used in children, the accuracy of the AED rhythm analysis algorithms should be validated with rhythms obtained from children aged 1-8 years.

The differences of pediatric vs. adult ECG rhythms have been quantified in a few studies so far, giving evidence about faster heart rates [2-5], shorter QRS durations [6], lower amplitudes and conduction velocities [2] for pediatric non-shockable rhythms. Aramendi et al. [5] reported significant changes of two morphological features (TCI, CM) associated with performance drop in pediatric non-shockable rhythms. Indeed, this trend was not observed for spectral features (A2, VFleak) as well as for pediatric shockable rhythms.
This study has two goals - first, to validate the performance of a commercial AED with pediatric ECG rhythms recorded during out-of-hospital cardiac arrest (OHCA) interventions, complying with the ERC guidelines. Second, to compare larger set of behavioral ECG characteristics in adults vs. pediatrics and to rank their robustness in the scenario where discrimination thresholds are trained for adults and applied on pediatrics.

\section{ECG Databases}

Adult and pediatric OHCA databases collected with a commercial AED (Fred Easy, Schiller Médical, France) in the region of Paris and suburban were analysed. The recorded ECGs were sampled at $500 \mathrm{~Hz}$, filtered (1-30 $\mathrm{Hz}$ ) by the AED's input hardware circuits for baseline drift and high-frequency noise suppression. The rhythm in 10s strips during AED analysis on noise-free ECGs was annotated by three experts as normal sinus rhythm (NSR), other non-shockable rhythm (ONR), asystole (ASYS), ventricular fibrillation (VF) in both databases:

(1) Adult DB (2011) from 742 patients, including 3690 ECG strips: 154 NSR, 1063 ONR, 2252 ASYS, 221 VF.

(2) Pediatric DB (2010-2014) from 191 children (6 years median age), including 881 ECG strips: 39 NSR, 294 ONR, 508 ASYS, 40 VF.

The defi-pads were placed in antero-lateral position (adults) and mainly antero-posterior (pediatrics).

All rhythms were almost equally distributed between pediatric and adult databases (Figure 1), representing the observed real-life OHCA scenario for rhythm incidence.

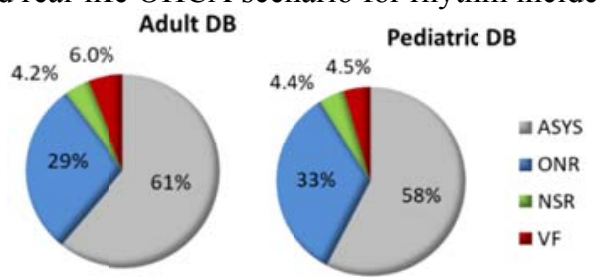

Figure 1. Incidence of rhythms in OHCA databases. 


\section{Methods}

\subsection{AED shock advisory system}

The study evaluated a shock advisory system (SAS) of a commercial AED (Fred Easy, Schiller Médical, France) with settings validated for adults [8]. The SAS shock/noshock decision was taken by analysis of 10s ECG strips relying on 8 basic ECG features:

- 4 morphological features of significant ECG peaks, including QRS complexes and VF waves: heart rate ( $\underline{\mathrm{HR}})$, peak-to-peak signal amplitude (SA), period uniformity $(\underline{\text { PeriodU }})$ and slope uniformity $(\underline{\text { SlopeU) }})$ of positive vs. negative peaks.

- 4 morphological features at the output of a narrow bandpass (BP) filter adjusted for QRS enhancement [9]: HR and amplitude of significant peaks (bpHR, bpSA), signal extrema (bpSE) $>25 \%$ bpSA, deflections from signal mean (bpSM) within the mean deviation band.

\subsection{Additional ECG features}

Five basic ECG descriptors which could be used for comprehensive analysis of waveform changes in children vs. adults were adopted from published studies:

- CVEL: Conduction velocity introduced in [2] as the rate of ventricular activation. CVEL measures the slope of the first major QRS deflection or the steepest VF wave.

- Leak: Leakage [10] evaluating the deviation of the ECG waveform from a sinusoid with the mean rhythm frequency. It was calculated by summing ECG with its copy shifted by a half a period, known as VF-filter.

- 3 power spectral density (PSD) features [5], evaluating:

- $\quad$ f0: the dominant frequency at maximal PSD;

- $\quad$ Pf0: PSD proportion within fixed band $\mathrm{f0} \pm 1.35 \mathrm{~Hz}$;

- $\quad$ varF: frequency variance as the PSD dispersion from the mean frequency in a band $2.5-14 \mathrm{~Hz}$.

- A2 [11]: ratio of the amplitude spectrum area in a band $0.7 \mathrm{f0}-1.4 \mathrm{f0}$ to the total amplitude spectrum area $>0.5 \mathrm{~Hz}$.

\section{Results and discussion}

Sensitivity (Se) and specificity (Sp) of SAS in AED was compared on pediatric vs. adult databases (Table 1). Se improvement $(+4.1 \%)$ and $\mathrm{Sp}$ drop in children was observed - maximal for NSR $(-2.6 \%)$ and negligible for ONR $(-0.2 \%)$ and ASYS $(-0.1 \%)$. The reason for such accuracy differences could be justified by analysis of basic ECG feature dependencies. We found significant correlations for numerous ECG features to $\mathrm{HR}$ in organized rhythms (OR), including (NSR+ONS) rhythms with QRS complexes (Figure 2). Therefore, we further consider 3 groups of HR dependent OR (>100 bpm, 60$100 \mathrm{bpm},<60 \mathrm{bpm})$. As shown in Table 1, accuracy drop in children was observed only for OR $>100 \mathrm{bpm}(-2 \%)$.
Table 1. Prospectively recorded SAS performance for different rhythms in adult and pediatric OHCA databases.

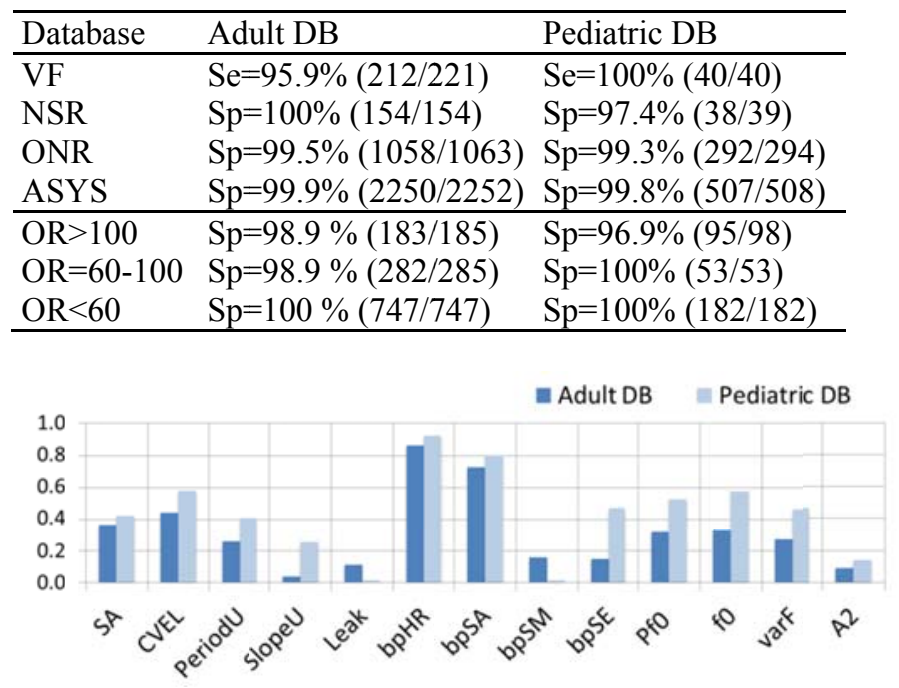

Figure 2. Correlation of ECG features to HR for OR.
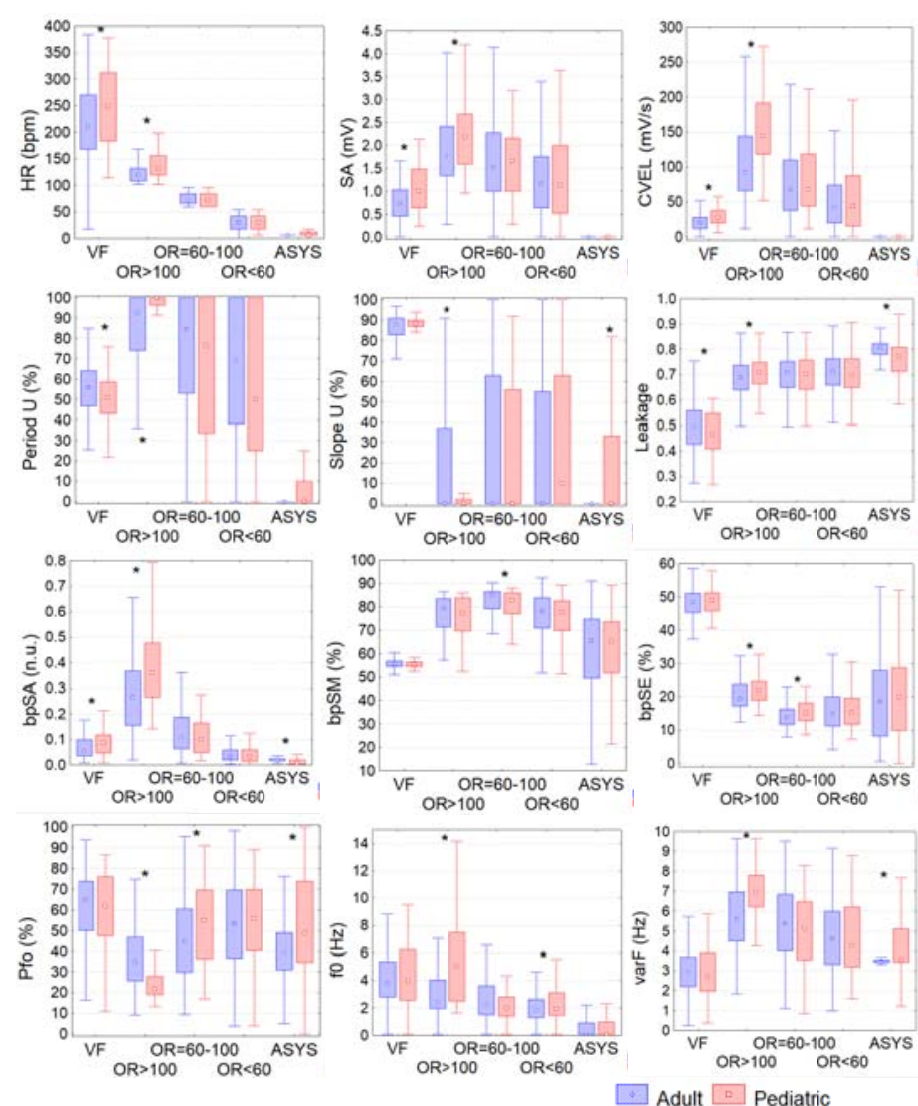

Figure 3. Box-plot distributions (median value, interquartile range, non-outlier range) of different ECG features. $* p<0.05$ : Significant differences of adult vs. pediatric rhythms by Mann-Whitney U-test. 
Table 2. Median value differences (Pediatric DB -Adult DB) of all ECG features for specific rhythms (VF, OR, ASYS). ${ }^{*} \mathrm{p}<0.05$ : significant differences between adult vs. pediatric ECG characteristics by Mann-Whitney U-test.

\begin{tabular}{|c|c|c|c|c|c|c|c|c|c|c|c|c|c|c|}
\hline & $\begin{array}{c}\mathrm{HR} \\
(\mathrm{bpm})\end{array}$ & $\begin{array}{c}\text { SA } \\
(\mathrm{mV})\end{array}$ & $\begin{array}{l}\text { CVEL } \\
(\mathrm{mV} / \mathrm{s})\end{array}$ & $\begin{array}{c}\text { PeriodU } \\
(\%)\end{array}$ & $\begin{array}{c}\text { SlopeU } \\
(\%)\end{array}$ & $\begin{array}{l}\text { Leak } \\
\text { (n.u.) }\end{array}$ & $\begin{array}{l}\text { bpHR } \\
\text { (bpm) }\end{array}$ & $\begin{array}{l}\text { bpSA } \\
\text { (n.u) }\end{array}$ & $\begin{array}{c}\text { bpSM } \\
(\%)\end{array}$ & $\begin{array}{c}\text { bpSE } \\
(\%)\end{array}$ & $\begin{array}{l}\text { Pfo } \\
(\%)\end{array}$ & $\begin{array}{c}\text { f0 } \\
(\mathrm{Hz})\end{array}$ & $\begin{array}{l}\operatorname{varF} \\
(\mathrm{Hz})\end{array}$ & $\begin{array}{c}\text { A2 } \\
\text { (n.u.) }\end{array}$ \\
\hline$\overline{\mathrm{VF}}$ & $39^{*}$ & $.26^{*}$ & $8 *$ & $-5^{*}$ & 1.5 & $-.033^{*}$ & 0 & $.037 *$ & -0.14 & 0.64 & -3.0 & 0.31 & -0.24 & $.054^{*}$ \\
\hline $\mathrm{OR}>100$ & $12 *$ & $.40^{*}$ & $52^{*}$ & $7.5^{*}$ & $0^{*}$ & $.022 *$ & $12^{*}$ & $.093 *$ & -2.12 & $2.5^{*}$ & $-13^{*}$ & $2.63 *$ & $1.34^{*}$ & -.017 \\
\hline $\mathrm{OR}=60-100$ & 0 & .14 & 0 & -8 & 0 & -.007 & 6 & -.008 & $-2.08 *$ & $1.4 *$ & $10.2 *$ & -0.24 & -0.24 & .009 \\
\hline $\mathrm{OR}<60$ & 0 & -.03 & 2 & -19 & 10 & -.014 & 0 & 0 & -0.44 & 0.46 & 2.5 & $0.09 *$ & -0.37 & .000 \\
\hline ASYS & 0 & 0 & 0 & $0^{*}$ & $0^{*}$ & $-.034 *$ & $24 *$ & $-.014 *$ & -0.32 & 1.4 & $9.4 *$ & $-0.06^{*}$ & $0.12 *$ & .002 \\
\hline
\end{tabular}

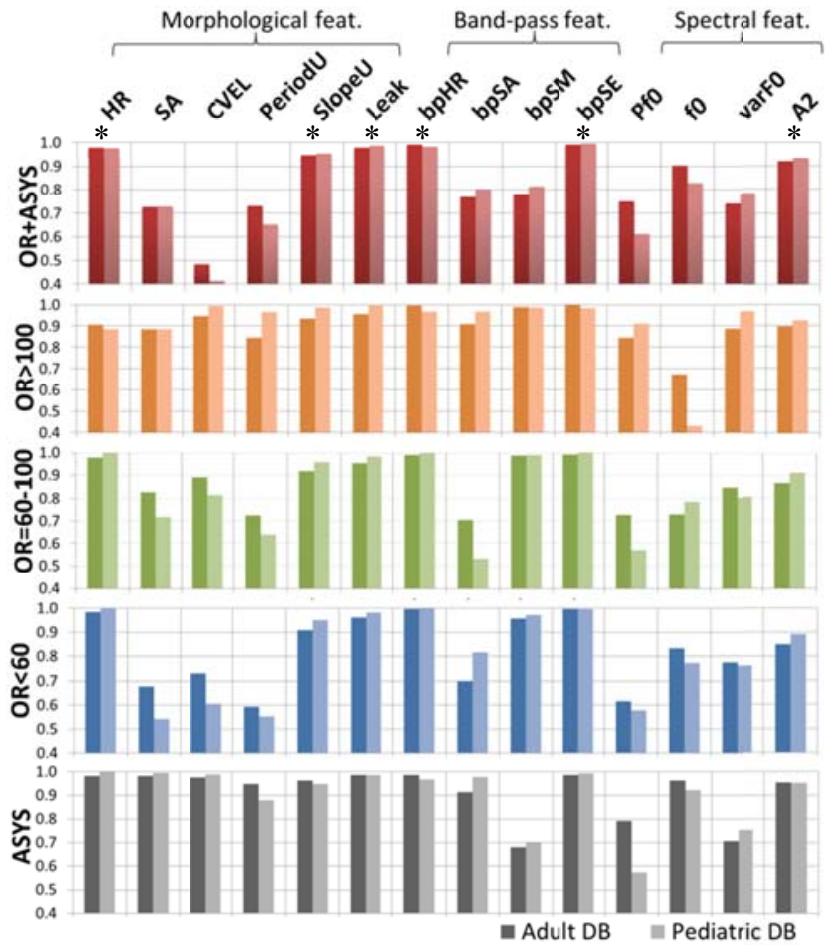

Figure 4. AUC training (Adult DB) and AUC test (Pediatric DB) of different ECG features, estimated by LDA classifier of VF vs. specific non-shockable rhythms.

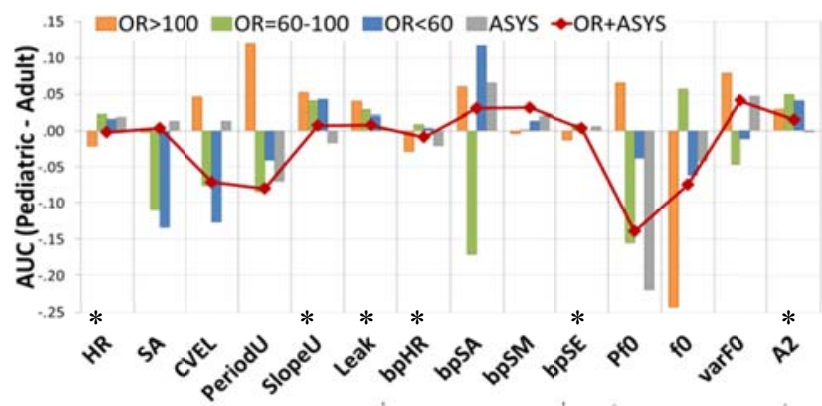

Figure 5. AUC difference (Pediatric-Adult) shows performance degradation (negative value) or improvement (positive value) when ECG features are tested on the pediatric DB for detection of VF vs. non-shockable rhythms.

Marks '*' highlight 6 features with the best AUC (Figure 4) without significant AUC degradation (Figure 5).
The statistical distributions of the ECG features for adult and pediatric databases (Figure 3) were compared by the non-parametric Mann-Whitney U-test, and the median value differences were reported in Table 1 . They showed that adult and pediatric rhythms presented certain significant differences $(\mathrm{p}<0.05)$ :

- VF: 7 feats (HR, SA, CVEL, PeriodU, Leak, bpSA, A2), indicating altered morphology of pediatric VF in terms of significantly more rapid, higher in amplitude, higher in velocity, less periodical and more sinusoidal VF waves.

- $\mathrm{OR}>100$ bpm: 12 features (all except bpSM, A2), indicating that pediatric non-shockable tachycardia is: significantly more rapid and steep; has more periodical and less uniform slope of positive and negative peaks, with morphology tending to differ from a sinusoid; has higher amplitude peaks, also seen well at the BP filter output for QRS enhancement; the spectrum is wider, with higher dominant frequency and presence of harmonics.

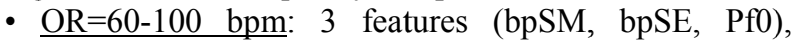
showing that only a few frequency dependent features are significantly altered in pediatrics, related to narrow-band PSD around the dominant frequency and fewer lowamplitude deflections at the BP QRS filter output.

- $\underline{\mathrm{OR}<60 \mathrm{bpm}}$ : 1 feature (f0), related to the slight rise of the dominant frequency $(+0.09 \mathrm{~Hz})$ in pediatric slow OR.

- ASYS: 6 features (Leak, bpHR, bpSA, Pf0, f0, varF) indicating alteration of the frequency content of the lowamplitude signal deflections during pediatric ASYS.

We contradict the finding for significantly lower CVEL and SA in pediatric OR [2], noting the different placement of defi pads in OHCA: mainly antero-posterior (pediatrics) vs. antero-lateral (adults).

The observed rhythm-specific changes of the pediatric ECG characteristics might have positive or negative effect on the safety of the VF detection algorithm in children that has been trained with thresholds adjusted for adults. This effect was evaluated with a standard linear discriminant analysis (LDA), whose discriminant function was trained to make binary classification of adult rhythms: VF vs. OR, VF vs. ASYS, VF vs. all nonshockable (OR+ASYS). The test of the same discriminant function with rhythms from the pediatric DB was reported in terms of area under the receiver operating characteristic curve (AUC ROC) in Figure 4. Figure 5 quantifies the difference between training and test AUC. 
Our global evaluation highlighted 6 features $\left({ }^{*} '\right)$ : morphology (HR, SlopeU, Leak), BP filter (bpHR, bpSE), spectral (A2), providing the most powerful AUC $>0.9$ and the most robust AUC(Pediatric-Adult $)>-0.025$. Two features (Leak, A2) were also confirmed as robust in [7].

All other features presented certain deficiencies for specific rhythms with remarkable pediatric AUC drop by $0.1-0.25$ observed in Figure 5, i.e.: spectral features f0 (OR $>100)$, Pf0 (all OR $<100$, ASYS); morphological features SA, CVEL, PeriodU (all $\mathrm{OR}<100$ ); band-pass signal amplitude bpSA (OR=60-100).

Although, some noted significant differences between pediatric and adult rhythms, our results clearly indicate that they do not necessarily lead to SAS accuracy drop. It is possible to design one AED shock advisory algorithm, being equally safe for adults and pediatrics, if the embedded ECG analysis features and their synergistic combinations are carefully selected to not drop the accuracy on a reference pediatric dataset. Generally, the number of shockable cases in published pediatric intrahospital $(\mathrm{IH})$ datasets is small (Table 3 ) due to the low prevalence of VF/VT during cardiac arrest in children. This is the first study, which is analysing data from outof-hospital emergency interventions. Our database indicates that the relative VF prevalence among children is very similar to adults $(4.5 \%$ vs. $6 \%$, Figure 1$)$, however all pediatric $\mathrm{OHCA}$ interventions are 15 -fold less frequent than adults (742 adults/1 year vs. 191 children/4 years), consistent with the reports [1]. Therefore, a common limitation of this and all published studies is the use of multiple samples from single patients. A consensus on the creation of a public pediatric database with continuous aggregation from different sources would provide the

Table 3. Review on SAS rhythm analysis with pediatric databases: Se (VF, VT: ventr. tachycardia $>150 \mathrm{bpm}$ ), Sp (NSR, ONR, ASYS). The publicly reported number of ECG strips (duration $3 \mathrm{~s}-15 \mathrm{~s}$ ) is presented in brackets.

\begin{tabular}{lccccc}
\hline & VF & VT & NSR & ONR & ASYS \\
\hline Checchin et al & $96 \%$ & $71 \%$ & $100 \%$ & $100 \%$ & $100 \%$ \\
2001 [3] (IH) & $(46)$ & $(32)$ & $(173)$ & $(251)$ & $(39)$ \\
\hline Atkinson et al & $98.6 \%$ & $100 \%$ & $99.2 \%$ & $99.8 \%$ & $100 \%$ \\
2003 [12] (IH) & $(73)$ & $(3)$ & $(798)$ & $(595)$ & $(79)$ \\
\hline Atkins et al & $100 \%$ & $94.9 \%$ & $100 \%$ & $99.6 \%$ & $100 \%$ \\
2008 [4] (IH) & $(42)$ & $(78)$ & $(208)$ & $(348)$ & $(29)$ \\
\hline Irusta et al & $98.4 \%$ & $31.4 \%$ & $99.6 \%$ & $100 \%$ & \\
2008 [13] (IH) & $(62)$ & $(70)$ & $(540)$ & $(419)$ & \\
\hline Irusta et al & & $96.9 \%$ & & $98.4 \%$ & \\
2009 [5] (IH) & & $(66)$ & & $(322)$ & \\
\hline Irusta et al & $100 \%$ & $96.4 \%$ & $100 \%$ & $100 \%$ & \\
2012 [14] (IH) & $(12)$ & $(28)$ & $(175)$ & $(122)$ & \\
\hline Didon et al & & & $100 \%$ & $99.8 \%$ & \\
2010 [15] (IH) & & & $(4737)$ & $(5564)$ & \\
\hline This study & $100 \%$ & & $97.4 \%$ & $99.3 \%$ & $99.8 \%$ \\
(OHCA) & $(40)$ & & $(39)$ & $(294)$ & $(508)$ \\
\hline
\end{tabular}

ground for valid AED performance reports on reasonable sample sizes in all rhythm categories.

\section{References}

[1] Maconochie IK, Bingham R, Eich C, et al. European Resuscitation Council Guidelines for Resuscitation 2015. Section 6. Paediatric life support. Resus 2015;95:223-48.

[2] Rustwick B, Atkins D. Comparison of electrocardiographic characteristics of adults and children for automated external defibrillator algorithms. Pediatr Emer Care 2014;30:851-5.

[3] Cecchin F, Jorgenson D, Berul C, et al. Is arrhythmia detection by automatic external defibrillator accurate for children? Circulation 2001;103:2483-8.

[4] Atkins L, Scott W, Blaufox A, et al. Sensitivity and specificity of an automated defibrillator algorithm designed for pediatric patients. Resuscitation 2008;76:168-74.

[5] Irusta U, Ruiz J. An algorithm to discriminate supraventricular from ventricular tachycardia in automated external defibrillators valid for adult and paediatric patients. Resuscitation 2009; 80: 1229-33.

[6] Sharieff GQ, Rao SO. The pediatric ECG. Emerg Med Clin North Am 2006;24:195-208.

[7] Aramendi E, Irusta U, Pastor E, Bodegas A, Benito F. ECG spectral and morphological parameters reviewed and updated to detect adult and pediatric life-threatening arrhythmia. Physiol Meas 2010;31:749-61.

[8] Jekova I, Krasteva V, Ménétré S, et al. Bench study of the accuracy of a commercial AED arrhythmia analysis algorithm in the presence of electromagnetic interferences. Physiol Meas 2009;30:695-705.

[9] Jekova I, Krasteva V. Real time detection of ventricular fibrillation and tachycardia. Physiol Meas 2004;25:1167-78.

[10] Kuo S, Dillman R. Computer detection of ventricular fibrillation. Comp in Cardiol 1978; 347-9.

[11] Barro S, Ruiz R, Cabello D, Mira J. Algorithmic sequential decision-making in the frequency domain for life threatening ventricular arrhythmias and imitative artefacts: a diagnostic system J Biomed Eng 1989;11:320-8.

[12] Atkinson E, Mikysa B, Conway J, et al. Specificity and sensitivity of automated external defibrillator rhythm analysis in infants and children. Ann Emerg Med 2003;42(2):185-96.

[13] Irusta U, Ruiz J, Ruiz de Gauna S, Aramendi E. A pediatric shock advice algorithm based on the regularity of the detected beats. Comp in Cardiol 2008; 35:1033-6.

[14] Irusta U, Ruiz J, Aramendi E, Ruiz de Gauna S, Ayala U, Alonso E. A high-temporal resolution algorithm to discriminate shockable from nonshockable rhythms in adults and children. Resus 2012;83:1090-7.

[15] Didon JP, Jekova I, Krasteva V. Evaluation of a shock advisory system with non-shockable pediatric rhythms. Comp in Cardiol 2010; 37:525-8.

Address for correspondence.

Vessela Krasteva

Institute of Biophysics and Biomedical Engineering Acad. G. Bonchev str., bl.105, 1113, Sofia, Bulgaria vessika@biomed.bas.bg 